\title{
Update of the ULtra-early TRranexamic Acid after Subarachnoid Hemorrhage (ULTRA) trial: statistical analysis plan
}

\author{
René Post ${ }^{1} \mathbb{D}$, Menno R. Germans ${ }^{2}$, Bert A. Coert ${ }^{1}$, Gabriël J. E. Rinkel ${ }^{3}$, W. Peter Vandertop ${ }^{1}$ and Dagmar Verbaan ${ }^{1 *}$
}

\begin{abstract}
Background: Recurrent bleeding from an intracranial aneurysm after subarachnoid hemorrhage $(S A H)$ is associated with unfavorable outcome. Recurrent bleeding before aneurysm occlusion can be performed occurs in up to one in five patients and most often happens within the first $6 \mathrm{~h}$ after the primary hemorrhage. Reducing the rate of recurrent bleeding could be a major factor in improving clinical outcome after SAH. Tranexamic acid (TXA) reduces the risk of recurrent bleeding but has thus far not been shown to improve functional outcome, probably because of a higher risk of delayed cerebral ischemia (DCI). To reduce the risk of ultraearly recurrent bleeding, TXA should be administered as soon as possible after diagnosis and before transportation to a tertiary care center. If TXA is administered for a short duration (i.e., $<24 \mathrm{~h}$ ), it may not increase the risk of $\mathrm{DCl}$. The aim of this paper is to present in detail the statistical analysis plan (SAP) of the ULTRA trial (ULtra-early TRranexamic Acid after Subarachnoid Hemorrhage), which is currently enrolling patients and investigating whether ultraearly and short-term TXA treatment in patients with aneurysmal SAH improves clinical outcome at 6 months.

Methods/design: The ULTRA trial is a multicenter, prospective, randomized, open, blinded endpoint, parallel-group trial currently ongoing at 8 tertiary care centers and 16 of their referral centers in the Netherlands. Participants are randomized to standard care or to receive TXA at a loading dose of $1 \mathrm{~g}$, immediately followed by $1 \mathrm{~g}$ every $8 \mathrm{~h}$ for a maximum of $24 \mathrm{~h}$, in addition to standard care, as soon as SAH is diagnosed. In the TXA group, TXA administration is stopped immediately prior to treatment (coil or clip) of the causative aneurysm. Primary outcome is the modified Rankin Scale (mRS) score at 6 months after SAH, dichotomized into good (mRS 0-3) and poor (mRS 4-6) outcomes, assessed blind to treatment allocation. Secondary outcomes include case fatalities at 30 days and at 6 months and causes of poor clinical outcome. Safety outcomes are recurrent bleeding, DCl, hydrocephalus, perprocedural complications, and other complications such as infections occurring during hospitalization. Data analyses will be according to this prespecified SAP.
\end{abstract}

Trial registration: Netherlands Trial Register, NTR3272. Registered on 25 January 2012. ClinicalTrials.gov, NCT02684812. Registered on 17 February 2016.

Keywords: Subarachnoid hemorrhage, Intracranial aneurysm, Tranexamic acid, Clinical outcome, Recurrent bleeding, Statistical analysis plan, ULTRA

\footnotetext{
* Correspondence: d.verbaan@amsterdamumc.nl

${ }^{1}$ Department of Neurosurgery, Neurosurgical Center Amsterdam, Amsterdam

University Medical Centers, PO Box 22660, Amsterdam 1100 DD, the

Netherlands

Full list of author information is available at the end of the article
}

(c) The Author(s). 2020 Open Access This article is distributed under the terms of the Creative Commons Attribution 4.0 International License (http://creativecommons.org/licenses/by/4.0/), which permits unrestricted use, distribution, and reproduction in any medium, provided you give appropriate credit to the original author(s) and the source, provide a link to the Creative Commons license, and indicate if changes were made. The Creative Commons Public Domain Dedication waiver (http://creativecommons.org/publicdomain/zero/1.0/) applies to the data made available in this article, unless otherwise stated. 


\section{Background}

Subarachnoid hemorrhage (SAH) accounts for $5 \%$ of all strokes and has an incidence of 7.9 per 100,000 personyears [1]. Only $25 \%$ of all patients with aneurysmal SAH have a favorable outcome, and even then, most of these patients still have severe cognitive dysfunction and functional disabilities [2]. The case fatality rate in SAH is approximately $35 \%$ due to the initial hemorrhage or subsequent complications. A frequent complication and one of the major causes of death and disability is recurrent bleeding from the aneurysm, which occurs in $4-12 \%$ of patients who

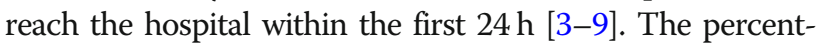
age of recurrent bleeding increases to $17 \%$ if cases of recurrent bleeding presenting within the first $6 \mathrm{~h}$ after the primary hemorrhage ("ultraearly recurrent bleeding") are also counted $[7,10]$. In daily clinical practice, aneurysm treatment is often postponed by either a delay in diagnosis or transfer to a tertiary treatment center [11-13]. Therefore, despite several efforts to improve the logistic processes, ultraearly recurrent bleeding still occurs before the aneurysm is secured. A strategy in addition to early aneurysm occlusion to reduce the number of recurrent bleedings is treatment with antifibrinolytic agents prior to aneurysm occlusion. Results from previous nonrandomized studies using early and short-term administration of antifibrinolytics showed reduction of recurrent bleeding without an increase in delayed cerebral ischemia (DCI) $[3,6,14,15]$. The only randomized controlled trial of early $(<48 \mathrm{~h})$ and short-term $(<72 \mathrm{~h})$ tranexamic acid (TXA) treatment confirmed a reduction in recurrent bleeding but did not assess the occurrence of DCI and was underpowered to show an effect on clinical outcome [3]. We therefore decided to perform a sufficiently powered randomized clinical trial in which TXA is administrated ultraearly (as soon as possible and at least within the first $24 \mathrm{~h}$ after the primary hemorrhage) and for an ultrashort time period $(<24 \mathrm{~h})$ to reduce the risk of the occurrence of DCI. The ULTRA (ULtra-early TRanexamic Acid after subarachnoid hemorrhage) trial is a multicenter, phase III, randomized, controlled, open-label, blinded endpoint trial performed in 8 tertiary care centers and 16 of their referral centers in the Netherlands (see Appendix for list of participants). We published the ULTRA trial protocol previously [16] and now describe the statistical analysis plan (SAP).

\section{Objectives}

The primary aim of the ULTRA trial is to evaluate whether ultraearly and short-term TXA treatment improves clinical outcome after 6 months in patients with SAH.

\section{Methods/design}

\section{Trial protocol development and conduct}

The ULTRA trial is registered with the Netherlands Trial Register (NTR3272; date of registration 25 January 2012) and ClinicalTrials.gov (2012-000343-26; registered on 17
February 2016). The ethics committee of the Amsterdam University Medical Centre (Amsterdam UMC, Amsterdam, the Netherlands) approved the trial protocol on 6 September 2012, starting with two treatment centers and one referral center. Six treatment centers and sixteen referral centers joined the study at a later date. The local accredited ethics committee of each participating hospital approved the local feasibility of the study protocol. During the course of the study, the accredited ethics committee approved three amendments with respect to changes in the inclusion and exclusion criteria. The study was conducted according to the principles of the Declaration of Helsinki, Dutch legislation regarding medical research involving human subjects [17-20], and good clinical practice (GCP) guidelines [21]. Because the majority of patients will not be able to give informed consent at admission, the informed consent procedure for this study is delayed in a so-called emergency procedure as described previously [16]. All study sites were monitored by an independent clinical research associate of the Amsterdam UMC Clinical Research Unit (Amsterdam, the Netherlands). An independent data and safety monitoring board (DSMB) monitored the study's progress, with a special focus on safety (see below). The trial will be reported according to the Consolidated Standards of reporting Trials (CONSORT) guidelines [22].

Inclusion and exclusion criteria are described in the previously published study protocol [16]. Adult patients with $\mathrm{SAH}$, diagnosed by noncontrast computed tomography (CT) within $24 \mathrm{~h}$ after the last hemorrhage, were included. During the trial, "no proficiency of the Dutch or English language" and "treatment for pulmonary embolism" were added to the exclusion criteria, whereas severe liver failure was removed from the exclusion criteria after consultation with the vascular internists. All changes were submitted to the accredited ethics committee as protocol amendments and were approved.

\section{Randomization and data collection}

Patients are randomly allocated in a 1:1 ratio to receive either ultraearly TXA treatment or standard care, stratified by treatment center. TXA is administered as a loading dose of $1 \mathrm{~g}$, immediately followed by $1 \mathrm{~g}$ every $8 \mathrm{~h}$ for a maximum of $24 \mathrm{~h}$, in addition to standard care, as soon as the SAH is diagnosed. In the TXA group, TXA administration is stopped immediately prior to treatment (coil or clip) of the causative aneurysm. To ensure allocation concealment, the randomization sequence was generated by using GCP-compliant ALEA $^{\circ}$ randomization software (ALEA Clinical, Abcoude, The Netherlands). Randomization was controlled in each treatment center and web-based, using a dedicated, password-protected, SSL-encrypted website. Data management was implemented according to GCP guidelines. Patients' data until hospital discharge and 6-month follow-up data are entered via an electronic case record form in a central 
GCP-compliant web-based database to facilitate on-site data entry (Oracle Clinical ${ }^{\oplus}$, Redwood Shores, CA, USA; OpenClinica LCC and collaborators, open source software, version 3.6, Waltham, MA, USA, www.OpenClinica.com and Castor Electronic Data Capture, Ciwit BV, Amsterdam, The Netherlands, 2018, www.castoredc.com). Security is guaranteed with login names, login codes, and encrypted data transfer.

\section{Primary outcome}

The primary outcome is clinical outcome at 6 months measured with the modified Rankin Scale (mRS) score by a standardized and validated telephone interview, performed by a trained research nurse who was blinded to treatment allocation [23, 24]. The mRS is dichotomized into good (mRS 0-3) and poor (mRS 4-6) outcomes [23, 25].

\section{Secondary outcomes}

Secondary outcomes include mRS score dichotomized into good (mRS scores $0-2$ ) and poor (mRS 3-6) outcomes, ordinal mRS score at 6 months, case fatality at 30 days and at 6 months, causes of poor outcome (directly related to primary $\mathrm{SAH}$, related to a complication of the $\mathrm{SAH}$, related to a complication of treatment, or related to another complication).

\section{Safety}

Safety outcomes were classified as follows:

1. Complications of SAH (recurrent bleeding, hydrocephalus, DCI)

2. Complications of treatment (per-procedural thromboembolic complication, infarct related to procedure, per-procedural rupture)

3. Other complications (extracranial thrombosis, deep venous thrombosis, pulmonary embolism), hemorrhagic complications, severe hyponatremia, pneumonia, meningitis, urinary tract infection, epilepsy, delirium, and Terson's syndrome

4. Suspected unexpected serious adverse reactions (SUSARs)

5. Other serious adverse events (SAEs)

Investigators recorded all SAEs during first hospital admission after ictus and reported any adverse event during first hospital admission after ictus that was not related to SAH. Although there are more secondary endpoints, this SAP will focus solely on the clinical (mRS scores and case fatalities) and safety (complications of SAH, complications of treatment, other complications, SUSARs, and other SAEs) secondary endpoints.

\section{Statistical methods specified in the study protocol Sample size calculation}

As described in the study protocol [16], the primary endpoint analysis of this study is based on the difference in percentage of patients with good outcome (mRS score 0 to 3) at 6 months after SAH between patients with and without TXA treatment. It is expected that TXA administration will increase the proportion of patients with a good outcome from $69 \%$ to $77.1 \%$.

This expected difference between the TXA and standard care groups was estimated using the results of renowned SAH studies and our own data (293 consecutive patients with aneurysmal SAH, added to angiogram-negative patients with SAH, treated at the AMC between 2008 and 2011). Of all patients with SAH who reach the hospital, 69\% have a good outcome (unpublished data). In our data, we find a recurrent bleeding rate of $17 \%$, which is consistent with numbers reported in previous studies (11-22\%) [3, 6, 8]. Among patients with recurrent bleeding, an estimated $20 \%$ will have a good outcome. Consequently, the percentage of patients with a good outcome without recurrent bleeding is $79 \%$. In the TXA group, the reduction in recurrent bleeding is expected to be $77 \%[3,6]$, which reduces the rate of recurrent bleeding to $3.9 \%$. Furthermore, TXA is anticipated to improve the percentage of good outcome in patients with recurrent bleeding from $20 \%$ to $30 \%$ [3]. Therefore, in the TXA group, 3.9\% will have recurrent bleeding, 30\% of whom will have a good outcome. A two-group chi-square test with a 0.05 two-sided significance level will have $80 \%$ power to detect the difference between a standard care group proportion of 0.69 and a treatment group proportion of 0.771 (odds ratio, 1.513) when the sample size in each group is 470 (940 patients in total). Taking some withdrawals into account, a total of 950 patients will be included.

\section{Originally proposed analyses}

In the previously published protocol [16], the originally proposed analyses are described, focusing on the intention-totreat (ITT) analysis. In the paragraphs below, the final and further detailed SAP is presented, including as-treated (AT) and per-protocol (PP) analyses because use of open-label TXA may modulate possible treatment effects.

\section{Interim analysis and safety reporting}

A DSMB was installed for this study to protect patients and advise the principal investigator on protecting the safety, validity, and credibility of the trial. Members include a clinically experienced neurologist/epidemiologist, an intensivist, and a statistician. The members were not involved in the trial and had no competing interests. The tasks, responsibilities, and working procedures of the DSMB were described in a charter.

The DSMB performed ongoing safety surveillance (every 6 months), especially with regard to the occurrence of SAEs in terms of increased ischemic events and serious extracranial thrombotic events, such as pulmonary embolism. Every 6 months, the DSMB receives a report prepared by an independent statistician that includes data by treatment group on primary outcome, predefined safety outcomes, other SAEs, and SUSARs. The DSMB also checks the assumptions for 
sample size calculations without performing statistical analysis. Additionally, the DSMB performed one interim analysis of unblinded effectiveness data during the study. This interim analysis was performed after inclusion of 475 patients in the trial to assess the strength of the efficacy data when half of the patients are enrolled.

The DSMB can recommend the steering committee of the ULTRA trial to:

- Adjust conduct, design, or sample size.

- Terminate the study prematurely when there is clear and substantial evidence of benefit

The justifications for a recommendation to terminate the study due to clear benefit will be based on prespecified stopping boundaries for the primary endpoint of the study (mRS score at 6 months). As a stopping rule, the Haybittle-Peto method [26, 27] will be used: interim efficacy analyses $(\mathrm{n}=475): P=0.001$; final efficacy analyses $(n=950): P=0.05$.

- Terminate the study prematurely when there is evidence of severe harm

The justifications for a recommendation to terminate the study due to clear harm will be based on data showing a notably increase of (serious) adverse events (including case fatalities) in the intervention group. No prespecified formal statistical stopping rule for safety is formulated.

- Terminate the study prematurely in case accrual rates are too low to provide adequate statistical power for identifying the primary endpoint.
If one or more of these situations occurs, the clinical relevance of the results will be incorporated into the decision whether to end the trial prematurely.

\section{Statistical analysis plan \\ Overall principles}

The database will not be unlocked until data regarding efficacy and safety from all patients have been included in the database after data verification and validation are performed and after the SAP has been submitted for publication. The data analysis will start after the 6-month follow-up data of the last included patient have been obtained. Analysis of the primary outcome will be performed according the ITT principle. Given the possible bias of open-label TXA treatment, primary outcome analysis will also be done in an AT population and a PP population to check the robustness of the main analysis, regardless of the presence of statistical significance in the overall analysis. Secondary outcomes will be analyzed in the ITT population, except for the main secondary outcome, mortality at 30 days and at 6 months, which will be analyzed in the ITT, AT, and PP populations. Safety outcomes will be analyzed in the ITT and AT populations. Statistical analyses will be done by the investigators of the ULTRA trial group (see Acknowledgements section). Statistical uncertainty will be expressed in a two-sided 95\% confidence interval (CI). Statistical analyses are performed using the IBM SPSS Statistics version 25 software (IBM Corporation, Armonk, NY, USA).

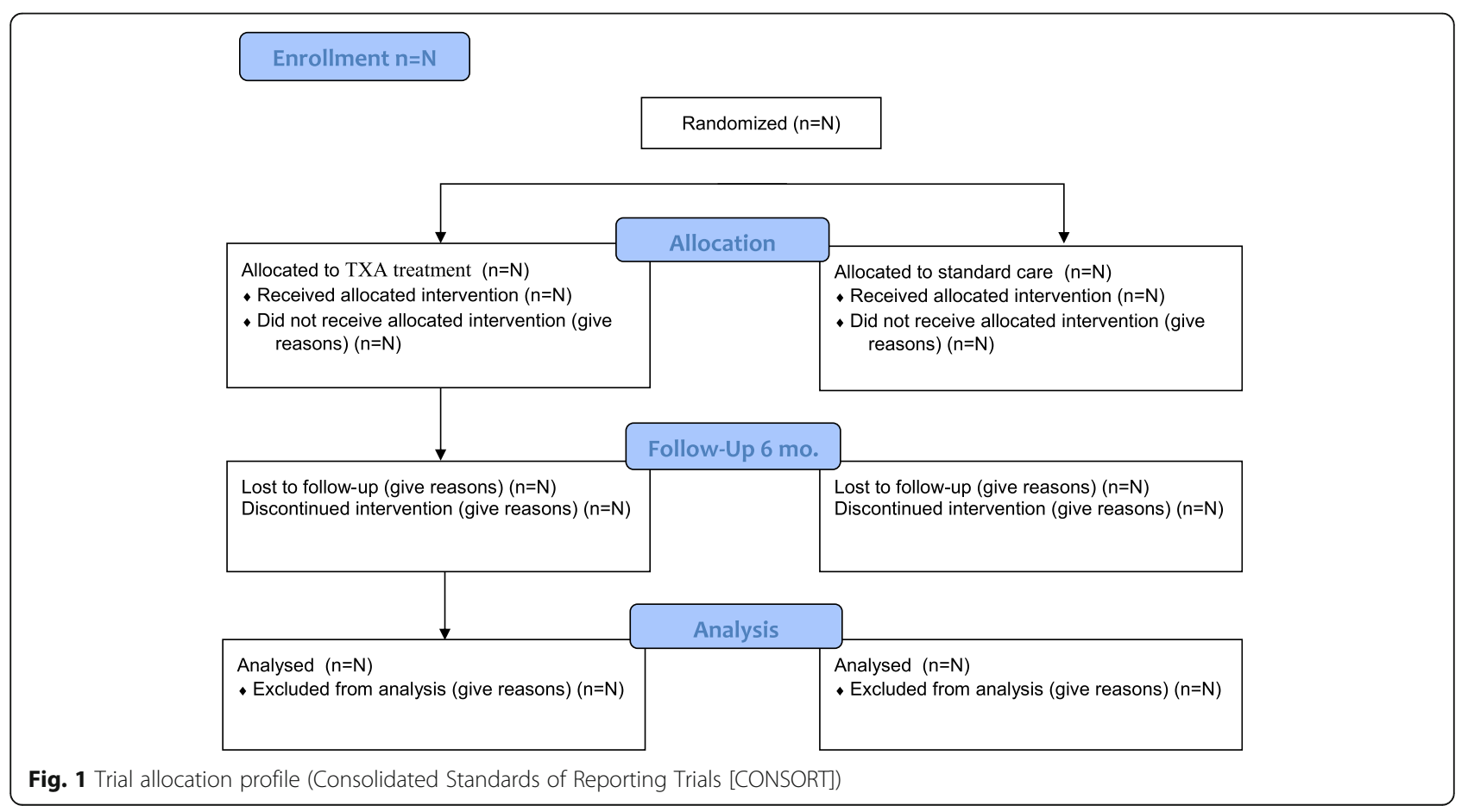




\section{Handling of missing data}

In case of missing data, every attempt will be made to retrieve the data. Because loss to follow-up is expected to be very low $(<1 \%$ missing data on the primary outcome), outcome data will not be imputed. We will state which data are missing and calculate frequencies using the total number of patients with available data. When a patient is lost to followup missing his/her 6-month mRS score, this patient cannot be included in the analysis of the primary outcome. If possible, these patients will be included in the secondary outcome analyses. When a patient has withdrawn consent, we will use all available data up until withdrawal of consent [28].

\section{Definition of analysis sets}

ITT population All randomized patients will be analyzed in the treatment group to which they were originally allocated, regardless of nonadherence or deviations from protocol (Table 5 in Appendix).

As-treated population Patients will be analyzed in groups according to treatment received, regardless of allocated treatment at randomization, thus creating a group that received at least one dose of TXA (intervention) and a group that did not (control). The patients will still be included in the AT analysis if there was a protocol violation (e.g., TXA administration not according to study protocol or not meeting inclusion or exclusion criteria).

Per-protocol population In the PP population, patients allocated to the standard care group who did not receive TXA will be included, as well as patients allocated to the TXA group who received TXA (at least one dose). The patients will still be included in the PP analysis if there was a protocol violation.

\section{Statistical analyses Patient flow}

The flow of participants will be displayed in the CONSORT flow diagram (Fig. 1), including the total number of randomized patients and then showing per treatment group the numbers receiving allocated treatment, withdrawing consent, and lost to follow-up.

\section{Protocol deviations}

When a patient is randomized but does not adhere to inclusion or exclusion criteria, this is considered a protocol deviation regarding eligibility. When a patient is allocated to the standard care group but does receive TXA, or when the patient is allocated to the TXA group but medication administration is not according to the protocol, this is considered a protocol deviation with respect to administration of medication. Protocol deviations will be line-listed in the Appendix.

\section{Baseline characteristics}

The baseline characteristics of all participants in each treatment group according to allocation will be outlined in a table without formal statistical testing. The table will describe the following variables: age, sex, World Federation of Neurosurgical Societies score, Fisher grade on noncontrast CT on initial (baseline) scan, medication use prior to SAH (antiplatelet therapy, anticoagulants, antihypertensive drugs), location of aneurysm, and treatment modality. Baseline variables will be summarized using simple descriptive statistics (Table 1). Continuous, normally distributed variables will be expressed as means and SDs; continuous, non-normally distributed, and ordinal variables will be expressed as medians (25th-75th percentiles); and categorical variables will be expressed

Table 1 Baseline characteristics of participants prior to randomization

\begin{tabular}{|c|c|c|}
\hline & $\begin{array}{l}\text { TXA group } \\
(n=X X X)\end{array}$ & $\begin{array}{l}\text { Standard care } \\
\text { group } \\
(n=X X X)\end{array}$ \\
\hline Age, yr, mean (SD) & NN.N (NN.N) & NN.N (NN.N) \\
\hline Female sex, $n(\%)$ & NNN (X) & NNN (X) \\
\hline \multicolumn{3}{|l|}{ WFNS grade } \\
\hline I, n (\%) & $N(X)$ & $N(X)$ \\
\hline$\|, n(\%)$ & $N(X)$ & $N(X)$ \\
\hline III, n (\%) & $N(X)$ & $N(X)$ \\
\hline IV, n (\%) & $N(X)$ & $N(X)$ \\
\hline$V, n(\%)$ & $N(X)$ & $N(X)$ \\
\hline \multicolumn{3}{|l|}{ Fisher grade } \\
\hline$\|, n(\%)$ & $N(X)$ & $N(X)$ \\
\hline III, n (\%) & $N(X)$ & $N(X)$ \\
\hline IV, n (\%) & $N(X)$ & $N(X)$ \\
\hline \multicolumn{3}{|l|}{ Medication prior to SAH } \\
\hline Platelet inhibitor, n (\%) & $N(X)$ & $N(X)$ \\
\hline Anticoagulation, $n(\%)$ & $N(X)$ & $N(X)$ \\
\hline Antihypertensive, $n$ (\%) & $N(X)$ & $N(X)$ \\
\hline None, $n(\%)$ & $N(X)$ & $N(X)$ \\
\hline \multicolumn{3}{|l|}{ Location of aneurysm } \\
\hline Anterior circulation, $n(\%)$ & $N(X)$ & $N(X)$ \\
\hline Posterior circulation, $n$ (\%) & $N(X)$ & $N(X)$ \\
\hline None, $n(\%)$ & $N(X)$ & $N(X)$ \\
\hline \multicolumn{3}{|l|}{ Treatment modality } \\
\hline Endovascular, n (\%) & $N(X)$ & $N(X)$ \\
\hline Clipping, n (\%) & $N(X)$ & $N(X)$ \\
\hline None, $n(\%)$ & $N(X)$ & $N(X)$ \\
\hline
\end{tabular}

SAH subarachnoid hemorrhage, TXA tranexamic acid, WFNS World Federation of Neurosurgical Societies

Data presented as mean (range), $n$ (\%), or median (IQR), unless noted otherwise 
Table 2 Primary outcome (modified Rankin Scale score at 6 months) and secondary outcomes

\begin{tabular}{lllll}
\hline & IT & & & \\
\cline { 2 - 5 } & TXA group & Standard care group & OR (95\% Cl) & aOR (95\% Cl) \\
\hline mRS 0-3 & $X X$ & $X X$ & $X X(X X-X X)$ & $X X(X X-X X)$ \\
Mortality at 30 days & $X X$ & $X X$ & $X X(X X-X X)$ & $X X(X X-X X)$ \\
Mortality at 6 mo & $X X$ & $X X$ & $X X(X X-X X)$ & $X X(X X-X X)$ \\
\hline
\end{tabular}

aOR adjusted odds ratio, $\mathrm{Cl}$ confidence interval, ITT intention to treat, $m R S$ modified Rankin Scale, OR odds ratio, TXA tranexamic acid

as counts and percentages. Normality of data will be explored by a normal Q-Q plot and tested by the ShapiroWilk test.

\section{Primary outcome}

The main statistical analysis will be based on the ITT principle. The occurrence of the primary outcome, dichotomized mRS score at 6 months (good versus poor as mRS 0 to 3 versus mRS 4 to 6 , respectively), will be compared between the two treatment groups (Table 2). The distribution of the mRS scores in both treatment groups will be depicted in a histogram (Fig. 2). Treatment effect will be expressed as a difference in proportions with corresponding 95\% CI and an odds ratio (OR) estimate with corresponding 95\% CI. Additionally, we will analyze the treatment effect on the dichotomized mRS score using multivariable logistic regression, adjusting for the stratification variable (treatment center) and, if necessary, clinically relevant baseline imbalances. Effect size will be expressed as an adjusted OR. The crude and adjusted analyses will also be performed in both the AT and PP populations.

\section{Sensitivity analyses}

Dichotomized mRS score is chosen as the primary outcome because results of the analysis are straightforward and easy to interpret. However, it is also clear that the cutoff is arbitrarily chosen and information is lost by dichotomization. Ordinal analysis of outcome data is becoming increasingly more common in acute stroke trials because it increases statistical power [29]:

Sensitivity analyses play a crucial role in assessing the robustness of the findings or conclusions based on primary analyses of data in clinical trials. They are a critical way to assess the impact, effect or influence of key assumptions or variations-such as different methods of analysis, definitions of outcomes, protocol deviations, missing data, and outliers-on the overall conclusions of a study [30].

Therefore, two sensitivity analyses will be performed: first, the dichotomized mRS using the cutoff frequently used in stroke (good outcome, mRS scores 0-2) will be analyzed using the same analysis as described by the primary outcome; second, the ordinal mRS score will be analyzed using an ordinal regression model on the total range of the mRS under the assumption of proportional odds (Table 3). If the assumption of ordinal regression does not hold, we will perform sliding dichotomy analysis [31].

When the loss to follow-up rate is $>10 \%$, a third sensitivity analysis will be performed. Data will be analyzed according to a worst case scenario; that is, patients lost to follow-up in the treatment group will have the worst possible outcome, and patients in the standard care group will have the best possible outcome.

\section{Secondary outcomes}

The secondary outcome analyses will compare case fatality at 30 days and at 6 months, causes of death or poor outcome at 6 months, and all safety outcomes between treatment groups (Table 4). The statistical analysis will also be based on the ITT principle. Treatment effect will be expressed in a difference in proportions with corresponding 95\% CI and an odds ratio (OR) estimate with corresponding $95 \% \mathrm{CI}$. The analyses for the main secondary outcome, mortality at discharge and at 6 months, will also be performed in both the AT and PP populations. The analyses

\begin{tabular}{|c|c|c|c|c|c|c|c|c|}
\hline \multirow[b]{2}{*}{ Standard Care $(\mathrm{n}=\mathrm{XXX})$} & mRS 0 & $\mathrm{mRS} 1$ & $\mathrm{mRS} 2$ & mRS 3 & $\mathrm{mRS} 4$ & mRS 5 & mRS 6 & \\
\hline & 10 & & 10 & 10 & 10 & 10 & 10 & 10 \\
\hline TXA $(n=X X X)$ & 10 & & 10 & 10 & 10 & 10 & 10 & 10 \\
\hline
\end{tabular}

Fig. 2 Distribution of $m R S$ at 6 months in the intention to treat analysis 
Table 3 Sensitivity analysis

\begin{tabular}{lllll}
\hline & \multicolumn{1}{l}{ IT } & & \\
\cline { 2 - 5 } & TXA group & Standard care group & OR (95\% Cl) & aOR (95\% Cl) \\
\hline EXcellent outcome (mRS 0-2) & $N N(X \%)$ & $N N(X \%)$ & $X . X X(X . X X-X . X X)$ & $X . X X(X . X X-X . X X)$ \\
Ordinal shift mRS) & $N N(X \%)$ & $N N(X \%)$ & & \\
mRS0 & & & \\
mRS1 & & & \\
mRS2 & & & \\
mRS3 & & & \\
mRS4 & & & \\
mRS5 & & & \\
mRS6 & & & \\
\hline
\end{tabular}

Data are $n(\%)$, mean (SD), or median (IQR)

$a O R$ adjusted odds ratio, $C l$ confidence interval, ITT intention to treat, $m R S$ modified Rankin Scale, OR odds ratio, TXA tranexamic acid

for the safety outcomes will also be performed in the AT population.

\section{Trial status}

Initially, two treatment centers started recruitment between July 2013 and February 2014, and six additional treatment centers started recruitment between April 2014 and September 2016. A total of 16 referral centers started recruitment between July 2013 and November 2018. All participating centers are in the Netherlands. Currently, we have enrolled all 955 patients.

Table 4 Safety outcomes during hospital admission

\begin{tabular}{|c|c|c|c|}
\hline & \multicolumn{3}{|l|}{$\mathrm{ITT}$} \\
\hline & TXA group $(n=x x)$ & Standard care group $(n=x x)$ & OR $(95 \% \mathrm{Cl})$ \\
\hline Any SAE, $n(\%)$ & $\mathrm{NN}(\mathrm{X})$ & $\mathrm{NN}(\mathrm{X})$ & $X . X X(X . X X-X . X X)$ \\
\hline Recurrent bleeding & $\mathrm{NN}(\mathrm{X})$ & $\mathrm{NN}(\mathrm{X})$ & $X . X X(X . X X-X . X X)$ \\
\hline Hydrocephalus & $\mathrm{NN}(\mathrm{X})$ & $\mathrm{NN}(\mathrm{X})$ & $X . X X(X . X X-X . X X)$ \\
\hline Delayed cerebral ischemia & $\mathrm{NN}(\mathrm{X})$ & $\mathrm{NN}(\mathrm{X})$ & $X . X X(X . X X-X . X X)$ \\
\hline $\begin{array}{l}\text { Thromboembolic complications during treatment } \\
\text { Coiling, } n(\%)\end{array}$ & $\mathrm{NN}(\mathrm{X})$ & $\mathrm{NN}(\mathrm{X})$ & $X . X X(X . X X-X . X X)$ \\
\hline $\begin{array}{l}\text { Infarct related to procedure } \\
\text { Clipping, } n(\%)\end{array}$ & $\mathrm{NN}(X)$ & $\mathrm{NN}(\mathrm{X})$ & $X . X X(X . X X-X . X X)$ \\
\hline $\begin{array}{l}\text { Procedural rupture } \\
\text { Coiling, } n(\%) \\
\text { Clipping, } n(\%)\end{array}$ & $\mathrm{NN}(\mathrm{X})$ & $\mathrm{NN}(\mathrm{X})$ & $X . X X(X . X X-X . X X)$ \\
\hline Extracranial thrombosis & $\mathrm{NN}(\mathrm{X})$ & $\mathrm{NN}(\mathrm{X})$ & $X . X X(X . X X-X . X X)$ \\
\hline - DVT & NN $(X)$ & $\mathrm{NN}(\mathrm{X})$ & $X . X X(X . X X-X . X X)$ \\
\hline$-P E$ & NN $(X)$ & NN $(X)$ & $X . X X(X . X X-X . X X)$ \\
\hline Hemorrhagic complication & NN $(X)$ & $\mathrm{NN}(X)$ & $X . X X(X . X X-X . X X)$ \\
\hline Severe hyponatremia & $\mathrm{NN}(\mathrm{X})$ & $\mathrm{NN}(\mathrm{X})$ & $X . X X(X . X X-X . X X)$ \\
\hline Pneumonia & $\mathrm{NN}(\mathrm{X})$ & $\mathrm{NN}(\mathrm{X})$ & $X . X X(X . X X-X . X X)$ \\
\hline Meningitis & $\mathrm{NN}(\mathrm{X})$ & $\mathrm{NN}(X)$ & $X . X X(X . X X-X . X X)$ \\
\hline Urinary tract infection & $\mathrm{NN}(\mathrm{X})$ & $\mathrm{NN}(\mathrm{X})$ & $X . X X(X . X X-X . X X)$ \\
\hline Epilepsy & $\mathrm{NN}(\mathrm{X})$ & $\mathrm{NN}(\mathrm{X})$ & $X . X X(X . X X-X . X X)$ \\
\hline Delirium & $\mathrm{NN}(\mathrm{X})$ & $\mathrm{NN}(\mathrm{X})$ & $X . X X(X . X X-X . X X)$ \\
\hline Terson's syndrome & $\mathrm{NN}(\mathrm{X})$ & $\mathrm{NN}(\mathrm{X})$ & $X . X X(X . X X-X . X X)$ \\
\hline SUSARS & $\mathrm{NN}(\mathrm{X})$ & $\mathrm{NN}(\mathrm{X})$ & $X . X X(X . X X-X . X X)$ \\
\hline Other & $\mathrm{NN}(\mathrm{X})$ & $N N(X)$ & $X . X X(X . X X-X . X X)$ \\
\hline
\end{tabular}

Cl confidence interval, DVT deep venous thrombosis, ITT intention to treat, OR odds ratio, PE pulmonary embolism, SAE serious adverse event, SUSARs suspected unexpected serious adverse reactions, TXA tranexamic acid 


\section{Appendix}

Table $\mathbf{5}$ Definition of population analysis sets

\begin{tabular}{|c|c|c|}
\hline Analysis population & TXA group & Standard care group \\
\hline Intention to treat ("as randomized") & $\begin{array}{l}\text { Patients randomized to TXA group: } \\
\text { - Including all protocol deviations }\end{array}$ & $\begin{array}{l}\text { Patients randomized to standard care group: } \\
\text { - Including all protocol deviations }\end{array}$ \\
\hline As treated ("actual treatment") & $\begin{array}{l}\text { Patients who received TXA (at least } \\
\text { one dose), regardless of allocated } \\
\text { treatment at randomization: } \\
\text { - Including patients who received } \\
\text { TXA as described according to protocol } \\
\text { - Including patients who received TXA } \\
\text { not following protocol (protocol deviation) } \\
\text { - Including patients with other protocol } \\
\text { deviations }\end{array}$ & $\begin{array}{l}\text { Patients who did not receive TXA, regardless } \\
\text { of allocated treatment at randomization: } \\
\text { - Including patients who received standard } \\
\text { care as described according to protocol } \\
\text { - Including patients who received standard } \\
\text { care not following protocol (protocol } \\
\text { deviation) } \\
\text { - Including patients with other protocol } \\
\text { deviations }\end{array}$ \\
\hline Per protocol & $\begin{array}{l}\text { Patients randomized to TXA group who } \\
\text { received TXA (at least one dose): } \\
\text { - Including patients who received TXA } \\
\text { as described according to protocol } \\
\text { - Including patients who received TXA } \\
\text { not following protocol (protocol deviation) } \\
\text { - Including patients with other protocol } \\
\text { deviations }\end{array}$ & $\begin{array}{l}\text { Patients randomized to standard care group } \\
\text { who did not receive TXA: } \\
\text { - Including patients who received standard } \\
\text { care as described according to protocol } \\
\text { - Including patients who received standard } \\
\text { care not following protocol (protocol deviation) } \\
\text { - Including patients with other protocol deviations }\end{array}$ \\
\hline
\end{tabular}

TXA tranexamic acid

\section{Abbreviations}

aOR: Adjusted odds ratio; AT: As treated; Cl: Confidence interval; CONSORT: Consolidated Standards of Reporting Trials; CT: Computed tomography; DCl: Delayed cerebral ischemia; DSMB: Data and safety monitoring board; DVT: Deep venous thrombosis; GCP: Good clinical practice; ITT: Intention to treat; mRS: Modified Rankin Scale; OR: Odds ratio; PE: Pulmonary embolism; PP: Per protocol; SAE: Serious adverse event; SAH: Subarachnoid hemorrhage; SAP: Statistical analysis plan; SUSAR: Suspected unexpected serious adverse reaction; TXA: Tranexamic acid; WFNS: World Federation of Neurosurgical Societies

\section{Acknowledgements}

Not applicable.

\section{ULTRA Trial Group}

Steering Committee

W.P. Vandertop

G.J. Rinkel

B.A. Coert

M.R. Germans

Y.B.W.E.M. Roos

R. van den Berg

C.B.L.M. Majoie

J. Horn

Principal Investigators of treatment Centers

D. Verbaan - Amsterdam UMC

G.J. Rinkel / M.D.I. Vergouwen - UMC Utrecht

H. Kieft - Isala

D. Nanda - Isala

F.C. de Beer - Isala

M.Voorend - Maastricht UMC

R.J.van Oostenbrugge - Maastricht UMC

P.W.A Willems - LUMC

R.W. Koot - LUMC

K. Jellema - HMC

J.F.C. Wolfs - HMC

C.M.A.A. Roks - ETZ

B. van der Pol - ETZ

H.D. Boogaarts - Radboud UMC

Principal Investigators of referral Centers

P.H.A. Halkes - Noordwest Ziekenhuisgroep

T.C. van der Ree - Dijklander Ziekenhuis

F. de Beer - Spaarne Gasthuis
M. de Graaf - MC Slotervaart

D.S.M. Molenaar - Ziekenhuis Amstelland

J. van de Vlekkert - Flevoziekenhuis

L.J.A. Reichman- ZGT

P.J.A.M. Brouwers - Medisch Spectrum Twente

J.R. de Kruijk - Tergooi

J.B.M. ten Holter - Deventer Ziekenhuis

H.P. Bienfait - Gelre ziekenhuizen

H.J.G. Dieks - Saxenburgh Groep

K. de Gans - Groene Hart Ziekenhuis

S.E. Vermeer - Rijnstate

V.I.H. Kwa - OLVG / R.M. van den Berg-Vos - OLVG

\section{Authors' contributions}

RP was responsible for study conception and design, acquisition of data, statistical analysis and interpretation of data, drafting the article, critically revising the article, reviewing the submitted version of the manuscript, and approval of the final version of the manuscript. MRG was responsible for study conception and design, critically revising the article, review of the submitted version of the manuscript, and approval of the final version of the manuscript. BAC was responsible for critically revising the article, reviewing the submitted version of manuscript, and approval of the final version of the manuscript. GJER was responsible for study conception and design, critically revising the article, reviewing the submitted version of the manuscript, and approval of the final version of the manuscript. WPV was responsible for study conception and design, critically revising the article, reviewing the submitted version of the manuscript, and approval of the final version of the manuscript. DV was responsible for study conception and design, critically revising the article, reviewing the submitted version of the manuscript, and approval of the final version of the manuscript.

\section{Funding}

The ULTRA trial was funded by Fonds Ohra (project number 1202-031).

\section{Availability of data and materials}

The datasets used and/or analyzed during the current study are available from the corresponding author on reasonable request.

Ethics approval and consent to participate

We obtained research ethics committee approval before patients were enrolled from the Academic Medical Centre Ethics Committee (MEC NL39577.018.12) in the Netherlands on 6 September 2012. The trial was 
conducted according to the principles of the Declaration of Helsinki and national laws, such as the Medical Research involving Human Subjects Act (WMO) in the Netherlands. Potential participants or their legal representatives were provided with written information in their native language explaining the trial, consequences of participation, and a statement that participation was voluntary and refusal to participate would not change medical treatment. Collaborating clinicians at each hospital site recruited participants and obtained written informed consent from the participants or their legal representatives.

\section{Consent for publication}

Not applicable.

\section{Competing interests}

The authors declare that they have no competing interests.

\section{Author details}

${ }^{1}$ Department of Neurosurgery, Neurosurgical Center Amsterdam, Amsterdam University Medical Centers, PO Box 22660, Amsterdam 1100 DD, the Netherlands. ${ }^{2}$ Department of Neurosurgery, Clinical Neuroscience Center, University Hospital Zurich, Frauenklinikstrasse 10, 8091 Zurich, Switzerland. ${ }^{3}$ Department of Neurology and Neurosurgery, Rudolf Magnus Institute of Neuroscience, University Medical Center Utrecht, PO Box 85060, Utrecht 3508 $A B$, the Netherlands.

\section{Received: 22 July 2019 Accepted: 29 January 2020}

\section{Published online: 18 February 2020}

\section{References}

1. Etminan N, Chang HS, Hackenberg K, de Rooij NK, Vergouwen MDI, Rinkel GJE, et al. Worldwide incidence of aneurysmal subarachnoid hemorrhage according to region, time period, blood pressure, and smoking prevalence in the population: a systematic review and meta-analysis. JAMA Neurol. 2019;76(5):588-97.

2. Al-Khindi T, Macdonald RL, Schweizer TA. Cognitive and functional outcome after aneurysmal subarachnoid hemorrhage. Stroke. 2010;41(8):e519-36.

3. Hillman J, Fridriksson S, Nilsson O, Yu Z, Saveland H, Jakobsson KE. Immediate administration of tranexamic acid and reduced incidence of early rebleeding after aneurysmal subarachnoid hemorrhage: a prospective randomized study. J Neurosurg. 2002;97(4):771-8.

4. Beck J, Raabe A, Szelenyi A, Berkefeld J, Gerlach R, Setzer M, et al. Sentinel headache and the risk of rebleeding after aneurysmal subarachnoid hemorrhage. Stroke. 2006;37(11):2733-7.

5. Brisman JL, Song JK, Newell DW. Cerebral aneurysms. N Engl J Med. 2006; 355(9):928-39.

6. Starke RM, Kim GH, Fernandez A, Komotar RJ, Hickman ZL, Otten ML, et al. Impact of a protocol for acute antifibrinolytic therapy on aneurysm rebleeding after subarachnoid hemorrhage. Stroke. 2008;39(9):2617-21.

7. Starke RM, Connolly ES. Rebleeding after aneurysmal subarachnoid hemorrhage. Neurocrit Care. 2011;15(2):241-6.

8. Guo LM, Zhou HY, Xu JW, Wang Y, Qiu YM, Jiang JY. Risk factors related to aneurysmal rebleeding. World Neurosurg. 2011;76(3-4):292-8 discussion 253-4.

9. Germans MR, Coert BA, Vandertop WP, Verbaan D. Time intervals from subarachnoid hemorrhage to rebleed. J Neurol. 2014;261(7):1425-31.

10. Fujii $Y$, Takeuchi S, Sasaki O, Minakawa T, Koike T, Tanaka R. Ultra-early rebleeding in spontaneous subarachnoid hemorrhage. J Neurosurg. 1996; 84(1):35-42.

11. Laidlaw JD, Siu KH. Ultra-early surgery for aneurysmal subarachnoid hemorrhage: outcomes for a consecutive series of 391 patients not selected by grade or age. J Neurosurg. 2002;97(2):250-8 discussion 247-9.

12. Lamb JN, Crocker M, Tait MJ, Bell BA, Papadopoulos MC. Delays in treating patients with good grade subarachnoid haemorrhage in London. $\mathrm{Br} J$ Neurosurg. 2011;25(2):243-8.

13. Robbert M, Germans MR, Hoogmoed J, van Straaten HA, Coert BA, Peter Vandertop $W$, et al. Time intervals from aneurysmal subarachnoid hemorrhage to treatment and factors contributing to delay. J Neurol. 2014; 261(3):473-9.

14. Gaberel T, Magheru C, Emery E, Derlon JM. Antifibrinolytic therapy in the management of aneurismal subarachnoid hemorrhage revisited: a metaanalysis. Acta Neurochir (Wien). 2012;154(1):1-9.
15. Baharoglu MI, Germans MR, Rinkel GJ, Algra A, Vermeulen M, van Gijn J, et al. Antifibrinolytic therapy for aneurysmal subarachnoid haemorrhage. Cochrane Database Syst Rev. 2013;(8):CD001245. https://doi.org/10.1002/ 14651858.CD001245.pub2

16. Germans MR, Post R, Coert BA, Rinkel GJ, Vandertop WP, Verbaan D. Ultraearly tranexamic acid after subarachnoid hemorrhage (ULTRA): study protocol for a randomized controlled trial. Trials. 2013;14:143.

17. World Medical Association (WMA). WMA Declaration of Helsinki - ethical principles for medical research involving human subjects. https://www.wma. net/policies-post/wma-declaration-of-helsinki-ethical-principles-for-medicalresearch-involving-human-subjects/. Accessed 15 Jan 2019.

18. Act of 26 February 1998, containing rules on medical research involving human subjects (Medical Research [Human Subjects] Act). https:/english. ccmo.nl/human-subjects. Accessed 15 Jan 2019.

19. Wet medisch-wetenschappelijk onderzoek met mensen http://wetten. overheid.nl/BWBR0009408/2017-03-01. Accessed 15 Jan 2019.

20. Federal Agency for Medicines and Health Products. De experimentenwet (7 mei 2004) en Uitvoeringsbesluit KB (koninklijk besluit) (30 juni 2004) https:// www.fagg-afmps.be/en/human_use/medicines/medicines/research_ development/clinical_trials. Accessed 15 Jan 2019.

21. International Conference on Harmonisation of Technical Requirements for Registration of Pharmaceuticals for Human Use (ICH). ICH harmonised tripartite guideline for good clinical practice E6(R1). 10 Jun 1996. http:// www.ich.org/fileadmin/Public_Web_Site/ICH_Products/Guidelines/Efficacy/ E6/E6_R1_Guideline.pdf. Accessed 15 Jan 2019.

22. Kessler KM. The CONSORT statement: explanation and elaboration [letter]. Ann Intern Med. 2002;136(12):926-7 author reply 926-7.

23. Janssen PM, Visser NA, Dorhout Mees SM, Klijn CJ, Algra A, Rinkel GJ. Comparison of telephone and face-to-face assessment of the modified Rankin Scale. Cerebrovasc Dis. 2010;29(2):137-9.

24. Savio K, Pietra GL, Oddone E, Reggiani M, Leone MA. Reliability of the modified Rankin Scale applied by telephone. Neurol Int. 2013;5(1):e2.

25. Rankin J. Cerebral vascular accidents in patients over the age of 60. II. Prognosis. Scott Med J. 1957;2(5):200-15.

26. Haybittle JL. Repeated assessment of results in clinical trials of cancer treatment. Br J Radiol. 1971;44(526):793-7.

27. Peto R, Pike MC, Armitage P, Breslow NE, Cox DR, Howard SV, et al. Design and analysis of randomized clinical trials requiring prolonged observation of each patient. I. Introduction and design. Br J Cancer. 1976;34(6):585-612.

28. Office of Medical Products and Tobacco, Office of Special Medical Programs, Office of Good Clinical Practice, U.S. Food and Drug Administration (FDA). Data retention when subjects withdraw from FDA-regulated clinical trials: guidance for sponsors, clinical investigators, and IRBs. Rockville: FDA; 2008

29. Optimising Analysis of Stroke Trials C, Bath PM, Gray L, Collier T, Pocock S, Carpenter J. Can we improve the statistical analysis of stroke trials? Statistical reanalysis of functional outcomes in stroke trials. Stroke. 2007:38(6):1911-5.

30. Thabane L, Mbuagbaw L, Zhang S, Samaan Z, Marcucci M, Ye C, et al. A tutorial on sensitivity analyses in clinical trials: the what, why, when and how. BMC Med Res Methodol. 2013;13:92.

31. Bath PM, Lees KR, Schellinger PD, Altman H, Bland M, Hogg C, et al. Statistical analysis of the primary outcome in acute stroke trials. Stroke. 2012;43(4):1171-8

\section{Publisher's Note}

Springer Nature remains neutral with regard to jurisdictional claims in published maps and institutional affiliations.

Ready to submit your research? Choose BMC and benefit from:

- fast, convenient online submission

- thorough peer review by experienced researchers in your field

- rapid publication on acceptance

- support for research data, including large and complex data types

- gold Open Access which fosters wider collaboration and increased citations

- maximum visibility for your research: over $100 \mathrm{M}$ website views per year

At $\mathrm{BMC}$, research is always in progress.

Learn more biomedcentral.com/submissions 